International Atomic Energy Agency

and

United Nations Educational Scientific and Cultural Organization

INTERNATIONAL CENTRE FOR THEORETICAL PHYSICS

\title{
HAMILTONIAN DESCRIPTION OF HIGHER ORDER LAGRANGIANS
}

\author{
M.S. Rashid and S.S. Khalil ${ }^{1}$
}

International Centre for Theoretical Physics, Trieste, Italy.

\begin{abstract}
Using Dirac's analysis of second class constraints, a Hamiltonian formulation of higher order Lagrangians is reached. The new Hamiltonian description recovers the conventional description of such systems due to Ostrogradsky, but unlike the later it provides a simpler structure for the phase space and consequently the underlying symplectic structure.
\end{abstract}

MIRAMARE - TRIESTE

December 1993

${ }^{1}$ On leave from: University of Ain Shams, Department of Mathematics, Cairo, Egypt. 


\section{Introduction}

In this paper we are going to investigate the phase space structure of certain class of dynamical systems which are oftenly ignored in the literature. These systems are described by higher order Lgrangians, which are not first order in the sense they contain higher order derivatives of the configuration space variables, for instance, second order or any other finite order say $m$ for that matter.

In order to reach to the Hamiltonian description of these systems one intuitively would like to follow the normal procedure used for the Hamiltonian description of ordinary systems (described by first order Lagrangians). Of course, as it is well known, the corner stone in this descript: $p^{t}$ is the Legender transformation, which for a first order Lagrangian $\mathcal{L}\left(q_{n}, \dot{q}_{n}\right)$ and the degrees of freedom it entails i.e. the configuration space $\mathrm{CO}$ - ordinates $q_{n}$ and their conjugate momenta, which are defined as

$$
P^{n}=\frac{\partial \mathcal{L}}{\partial \dot{q}_{n}}
$$

is given by

$$
\mathcal{H}=\sum_{n=1} p^{n} q_{n}-\mathcal{L}
$$

However for systems described by higher order Lagrangian one cannot blindly use the above expression for the Legender transformation, eq(2), as it is, since the resulting Hamiltonian does not produce the right equations of motion, rather one ought to seek an appropriate generalisation of the above transformation and check whether the equations of motion derived from the Hamiltionian-to-be agrees with those obtained by methods of variation. This very problem was considerer by Ostrogradsky a century ago [1],[2], in his analysis he considers systems described by the Lagrangian $\mathcal{L}\left(x, \dot{x}, \ddot{x}, \ldots, x^{(m)}\right)$, which is a function of the co-ordinates and their time derivatives up to a finite order say $m$. Then he showed that the equation of motion obtained by methods of variation is given by the expression

$$
\frac{\partial L}{\partial x}-\frac{d}{d t} \frac{\partial L}{\partial \dot{x}}+\ldots \ldots+(-1)^{m} \frac{d^{m}}{d t^{m}}\left\{\frac{\partial L}{\partial x^{m}}\right\} .
$$


To establish the Hamiltonian description of the above higher order Lagrangian, he first defined some generalised momenta as

$$
\begin{aligned}
& p^{1}=\frac{\partial L}{\partial \dot{x}}-\frac{d}{d t}\left\{\frac{\partial L}{\partial \ddot{x}}\right\}+\ldots . .+(-1)^{m-1} \frac{d^{m-1}}{d t^{m-1}}\left\{\frac{\partial L}{\partial x^{m}}\right\} \\
& p^{2}=\left\{\frac{\partial L}{\partial \ddot{x}}\right\}+\ldots .+(-1)^{m-2} \frac{d^{m-2}}{d t^{m-2}}\left\{\frac{\partial L}{\partial x^{m}}\right\} \\
& \vdots \\
& P^{m}=\frac{\partial L}{\partial x^{m}} .
\end{aligned}
$$

The new generalised momenta, eqs (4), are then used to generalised the Legender transformation which, in terms of these momenta, has the following simple form

$$
\mathcal{H}=p_{1} \dot{x}+p_{2} \ddot{x}+\ldots \ldots \ldots+p_{m} x^{(m)}-\mathcal{L} .
$$

With the Hamiltonian $\mathcal{H}$ at our disposal, it is a matter of few lines of algebra to show that the equations of motion derived from the above Hamiltonian are identical to the ones obtained by applying methods of variation to the Lagrangian $\mathcal{L}$. With this Ostrogradsky concluded his Hamiltonian description of such systems.

From the point of view of quantization the phase space structure of Ostrogradsky Hamiltionian description is quite intricate. Consequently the underlying symplectic geometry has a complicated structure, the thing makes any attempt to quantize these systems hindered by technical difficulties. Motivated by finding an alternate Hamiltonian description which is simpler in principle and gives a relatively simpler phase space structure, which makes the canonical quantization of higher order Lagrangian easier to carry out.

Our procedure is based on introducing a new degrees of freedom called auxiliary fields $q_{n}$, as well one has to introduce a set of constraints $\chi_{n}$ such that the number of constraints equals the number of auxiliary fields. In fact it turns out, as we are going to show in the next section, that all these constraints imposed on the extended phase space of the system are second class ones since the algebra of constraints does not vanish weakly on the surface of constraints i.e. $\left\{\chi_{n}, \chi_{m}\right\} \neq 0$. As a result the problem reduces to that of Hamiltonian description of a system with second class constraints. Such systems were extensively studied and a procedure for establishing a 
Hamiltonian description of which is explicitly given in refs [5],[6], [8] and[9]. For the sake of completeness, in the next few paragraphs we will give a brief account of Dirac's description of systems with second class constraints.

Acharactirization of constraints which is independent of the Lagrangian function used to describe the system was introduced by Dirac[5],[9]. A phase space quantity $R\left(q_{n}, p_{n}\right)$ is said to be first-class quantity if its poisson brackets with all constraints $\chi_{i}$ is weakly vanishing:

$$
\left\{R, \chi_{i}\right\} \leftrightarrow\left\{R, \chi_{i}\right\}=\sum_{k} c_{i}^{k} \chi_{k} \approx 0 .
$$

A quantity which is not first-class, namely which has a non weakly vanishing Poisson brackets with at least one of the constraints, is said to be secondclass. Therefore in terms of these notations, all the constraints are then classified as either first-class or second-class constraints.

In fact second class constraints appear whenever some phase space degrees of freedom are redundant for the description of the system. In principle these degrees of freedom can be solved for in terms of the other degrees of freedom. By discarding them, new Poisson brackets, referring to the remaining degrees of freedom are introduced. Such a procedure may be very difficult to implement in practice. However in our analysis of constraints we are going to use Dirac's approach which in principle solves the problem in all cases.

Let us denote all the second class constraints by $\chi_{i} \approx 0$. Then consider the matrix of Poisson brackets of second class constraints

$$
\Delta_{s s^{\prime}}=\left\{\chi_{s}, \chi_{s^{\prime}}\right\},
$$

such that $\Delta_{s s^{\prime}}$ is a regular matrix, and let the inverse be defined as

$$
C^{s s^{\prime}}=\left(\Delta^{-1}\right)^{s s^{\prime}}
$$

with

$$
\sum_{s^{\prime}} C^{s s^{\prime}}\left\{\chi_{s^{\prime}}, \chi_{s^{\prime \prime}}\right\}=\delta_{s^{\prime \prime}}^{s}
$$


The Dirac bracket of two phase space quantities is then given by

$$
\{f, g\}_{D}=\{f, g\}-\sum_{s, s^{\prime}}\left\{f, \chi_{s}\right\} C^{s s^{\prime}}\left\{\chi_{s^{\prime}}, g\right\} .
$$

It is easy to show that Dirac brackets are generalised Poisson bracket, in the sense that they satisfy the same properties as Poisson brackets except the values of the fundamental brackets which are modified.

The equation of motion of an arbitrary phase space quantity $f$, is given by

$$
\{f, \mathcal{H}\}_{D}=\{f, \mathcal{H}\}-\sum_{s^{\prime}, s^{\prime \prime}}\left\{f, \chi_{s^{\prime}}\right\} C^{s^{\prime} s^{\prime \prime}}\left\{\chi_{s^{\prime \prime}}, \mathcal{H}\right\}
$$

Moreover, Dirac bracket of an arbitrary phase space quantity $f$ with any second-class constraint $\chi_{s}$ is strictly zero i.e. $\left\{f, \chi_{s}\right\}_{D}=0$. This shows that Dirac bracket achieve precisely the modification of Poisson bracket, namely that Poisson brackets are redefined in such a way that the redundant degrees of freedom related to the second-class constraints $\chi_{s} \approx 0$ are effectively not included in the definition. As a result the second-class constraints can be imposed exactly, and not as weak equality, even before working out brackets, provided one uses Dirac brackets.

By using Dirac brackets and then solving the constraints ( this last step is not really necessary ) one obtains a description of the system in which all the redundant degrees of freedom related to the second-class constraints are consistently ignored.

This paper is organised as follows, in section two Dirac's analysis of constraints is applied to the alternative Lagranian, then the equivalence of the two Lgrangians is investigated. In section three we are going to compute the Dirac brackets of the phase space variables, since they lay at the heart of any quantization procedure. 


\section{Auxiliary Fields and Analysis of Con- straints}

In the previous section we have briefly given a Hamiltonian description of a class of dynamical systems described by higher order Lagrangians. But in this section we are going to show that on accounts of Dirac analysis of constraints and introducing some auxiliary fields one can establish an alternate, but simpler, Hamiltonian description of these systems.

The Construction proceeds, first by introducing the new auxiliary fields by making the following identification in the original Lagrangian $\mathcal{L}$

$$
q_{1}=\dot{x} ; \quad q_{2}=\dot{q}_{1} ; \ldots \ldots ; q_{m}=\dot{q}_{m-1} .
$$

Since the new auxiliary fields $q_{i}$ are not true physical degrees of freedom we will re-write the above equations as a set of $m$ constraints;

$$
\chi_{1}=\left(q_{1}-\dot{x}\right) \approx 0 ; \quad \chi_{2}=\left(q_{2}-\dot{q}_{1}\right) \approx 0 ; \ldots . ; \chi_{m}=\left(q_{m}-\dot{q}_{m-1}\right) \approx 0,
$$

in fact it is not hard to see that eqs(13) define the surface of constraints on the extended phase space. Consequently, one arrives to an alternate description based on the new Lagrangian;

$$
\mathcal{L}=\mathcal{L}_{0}\left(x, q_{1}, \ldots ., q_{m}, \dot{q_{m}}\right)+\sum \mu_{i} \chi_{i} .
$$

This Lagrangian, unlike the original Lagrangian, has the advantage of being first order in the sense it is only a function of the co-ordinates and their first time derivative.

Next in our procedure is to investigate the equivalence between the two descriptions at the Lagrangain level, by first deriving the equation of motion as a result of varying the co-ordinates of the extended phase space including the multipliers i.e. $\left(x, q_{i}^{\prime} s\right.$ and $\left.\mu_{i}^{\prime} s\right)$ and acquiring the new action, which is a functional of the new Lagrangian, eq (14), to be stationary. Then compare these equations with the original equation of motion eq(3). It is trivial to show that variations with respect to $x$ results in the following equation

$$
\dot{\mu}_{1}+\frac{\partial L}{\partial x}=0 \text {. }
$$


Similarly variations with respect to $q_{i}$ 's yield another set of equations

$$
\begin{aligned}
& \dot{\mu}_{2}+\mu_{1}+\frac{\partial L}{\partial q_{1}}=0 \\
& \vdots \\
& \dot{\mu}_{m}+\mu_{m-1}+\frac{\partial L}{\partial q_{m 1}}=0
\end{aligned}
$$

and

$$
\mu_{m}=\frac{\partial L}{\partial q_{m}}+\frac{d}{d t}\left(\frac{\partial L}{\partial \dot{q}_{m}}\right)
$$

and variations with respect to the multipliers reproduces the primary constraints

$$
\chi_{1}=0, \chi_{2}=0 ; \ldots \ldots . . \chi_{m}=0 .
$$

Removing the multipliers in eqs(15),(16)and (17) by successive substitution and using the constraints, eq(18), one obtains the expression

$$
\frac{\partial L_{0}}{\partial x}-\frac{d}{d t}\left(\frac{\partial L_{0}}{\partial \dot{x}}\right)+\frac{d^{2}}{d t^{2}}\left(\frac{\partial L_{0}}{\partial \ddot{x}}\right)+\ldots \ldots+(-1)^{m} \frac{d^{m}}{d t^{m}}\left(\frac{\partial L_{0}}{\partial x^{(m)}}\right)=0 .
$$

Which is identical to the original equation of motion as in the first description, the thing shows the equivalence between the two descriptions at the Lagrangian level.

In order to investigate the equivalence between the two descriptions at the Hamiltonian level, we are going to check whether the Hamiltonian-to-be reproduces the original equation of motion. To do so, we define the following set of canonical momenta

$$
\begin{aligned}
& P=\frac{\partial L}{\partial \dot{x}_{L}}=-\mu_{1}, \quad \Phi_{1}=P+\mu_{1} \approx 0, \\
& Q_{1}=\frac{\partial_{1}}{\partial q_{1}}=-\mu_{2}, \quad \Phi_{2}=Q_{1}+\mu_{2} \approx 0, \\
& \vdots \\
& Q_{m-1}=\frac{\partial L}{\partial q_{m-1}}=-\mu_{m}, \quad \Phi_{m}=Q_{m-1}+\mu_{m} \approx 0 .
\end{aligned}
$$

By observing that the conjugate momenta in eqs(20)are time independent, it follows immediately that they correspond to the first set of primary constraints in the new Hamiltonian description. In addition, the other set of 
primary constraints corresponds to the conjugate momenta of the multipliers;

$$
\Pi_{i}=\frac{\partial L}{\partial \dot{\mu}_{i}} \approx 0 \quad i=1, \ldots n .
$$

In fact it easy to show that the above constraints satisfy the following algebra

$$
\begin{aligned}
& \left\{\phi_{i}, \phi_{j}\right\}=0,\left\{\pi_{i}, \pi_{j}\right\}=0 \\
& \left\{\phi_{i}, \pi_{j}\right\}=\delta_{i j},
\end{aligned}
$$

which shows that these constraints are second-class constraints.

The form of the basic Hamiltonian $\mathcal{H}_{0}$ is

$$
\mathcal{H}_{0}=x P+\sum_{i=1}^{m} q_{i} Q^{i}+\sum_{i=1}^{m} \dot{\mu}_{i} \Pi^{i}-\mathcal{L}_{0}\left(x, q_{i}, \dot{q}_{m}\right)-\sum_{i=1}^{m} \mu_{i}\left(q_{i}-q_{i-1}\right),
$$

while the form the extended Hamiltonian $\mathcal{H}_{E}$ is

$$
\mathcal{H}_{E}=\mathcal{H}_{0}+\sum_{i=1}^{m} \lambda_{i} \Phi_{i}+\sum_{i=1}^{m} \lambda_{i}^{\prime} \Pi_{i}
$$

In this expression for the extended Hamiltonian, one determines the two arbitrary multipliers by acquiring the time evolution of the above constraints to vanish i.e. $\dot{\Phi}_{i}=\dot{\Pi}_{i}=0$. Observing that

$$
\begin{aligned}
& \left\{\Phi_{1}, \mathcal{H}_{0}\right\}=\frac{\partial L}{\partial z}+\dot{\mu_{1}} \\
& \left\{\Phi_{2}, \mathcal{H}_{0}\right\}=\frac{\partial L}{\partial q_{1}}+\dot{\mu_{2}} \\
& \vdots \\
& \left\{\Phi_{m}, \mathcal{H}_{0}\right\}=\frac{\partial L}{\partial q_{m 1}}+\dot{\mu_{m}}
\end{aligned}
$$

and

$$
\left\{\Pi_{i}, \mathcal{H}_{0}\right\}=\left(q_{i}-\dot{q}_{i-1}\right),
$$

then it follows trivially that

$$
\begin{aligned}
& \lambda_{i}=\left(q_{i}-\dot{q}_{i-1}\right) \\
& \lambda_{i}^{\prime}=-\frac{\partial L}{\partial q_{i-1}} \dot{\mu}_{i} .
\end{aligned}
$$


Substituting the value of the two multipliers in the expression for $\mathcal{H}_{E}$ gives the new expression

$$
\mathcal{H}_{E}=\mathcal{H}_{0}+\left(q_{i}-\dot{q}_{i-1}\right)\left(Q_{i-1}+\mu_{i}\right)-\frac{\partial L}{\partial q_{i-1}} \Pi^{i}-\dot{\mu}_{i} \Pi^{i}
$$

which after cancellations and rearrangements of some terms it has the new form

$$
\begin{aligned}
& \mathcal{H}_{E}=\dot{q}_{m} Q_{m}+\sum_{i=1}^{m} q_{i} Q_{i-1} \mathcal{L}_{0} \\
& \mathcal{H}_{E}=\dot{q}_{m} Q_{m}+q_{1} P+q_{2} Q_{1}+\ldots+q_{m} Q_{m-1}-\mathcal{L}_{0}
\end{aligned}
$$

To check the validity of the above Hamiltonian, let us derive the equations of motion, following the normal procedure of evaluating Poisson brackets of the phase space variables $\left(x, P, q_{i}, Q_{i}\right)$ with the extended Fiamiltonian $\mathcal{H}_{E}$. In doing so one gets the following equations

$$
\begin{aligned}
& \dot{P}=\left\{P, \mathcal{H}_{E}\right\}=\frac{\partial \mathcal{L}_{0}}{\partial x} \\
& \dot{Q}_{1}=\left\{Q_{1}, \mathcal{H}_{E}\right\}=-P+\frac{\partial \mathcal{L}_{0}}{\partial q_{1}} \\
& \dot{Q}_{2}=\left\{Q_{2}, \mathcal{H}_{E}\right\}=-Q_{1}+\frac{\partial \mathcal{L}_{0}}{\partial q_{2}} \\
& \vdots \\
& \dot{Q_{m}}=\left\{Q_{m}, \mathcal{H}_{E}\right\}=-Q_{m-1}+\frac{\partial \mathcal{L}_{0}}{\partial q_{m}},
\end{aligned}
$$

and

$$
\begin{aligned}
& \dot{x}=\left\{x, \mathcal{H}_{E}\right\}=q_{1} \\
& \dot{q}_{1}=\left\{q_{1}, \mathcal{H}_{E}\right\}=q_{2} \\
& \vdots \\
& \dot{q}_{m-1}=\left\{q_{m-1}, \mathcal{H}_{E}\right\}=q_{m} \\
& \dot{q}_{m}=\left\{q_{m}, \mathcal{H}_{E}\right\}=\dot{q}_{m} .
\end{aligned}
$$

The last equation in eqs $(31)$ is trivial, whereas the other equations are expected to appear since they are non other than the original constraints $\chi_{i}$ in the Lagrangian formulation. 
At this stage it is far from obvious whether the above equations reproduce the original equation of motion and expressions for the generalised momenta as the first description. To show that the above expressions for the generalised momenta are the same as those of the first description in disguise, one uses eqs(30) to obtain,

$$
P=-\dot{Q}_{1}+\frac{\partial L_{0}}{\partial q_{1}}
$$

followed by the observation,

$$
P=\frac{\partial L_{0}}{\partial q_{1}}-\frac{d}{d t}\left(\frac{\partial L_{0}}{\partial q_{2}}\right)+\ddot{Q}_{2}
$$

and then repeating this substitution $m$ times one finally gets

$$
P=\frac{\partial L_{0}}{\partial \dot{x}}-\frac{d}{d t}\left(\frac{\partial L_{0}}{\partial \ddot{x}}\right)-\frac{d^{2}}{d t^{2}}\left(\frac{\partial L_{0}}{\partial x^{3}}\right)+\ldots \ldots+(-1)^{m-1} \frac{d^{m-1}}{d t^{m 1}}\left(\frac{\partial L_{0}}{\partial x^{(m)}}\right) .
$$

Applying the same procedure to the rest of the momenta $Q_{1}, \ldots Q_{m}$, one arrives to the following expressions

$$
\begin{aligned}
& Q_{1}=\frac{\partial L_{0}}{\partial \ddot{x}}-\frac{d}{d t}\left(\frac{\partial L_{0}}{\partial x^{3}}\right)+\ldots \ldots . .+(-1)^{m-2} \frac{d^{m-2}}{d t^{m-2}}\left(\frac{\partial L_{0}}{\partial x^{(m)}}\right) \\
& \vdots \\
& Q_{m}=\frac{\partial L_{0}}{\partial x^{(m)}} .
\end{aligned}
$$

These equations are identical to the expressions for momenta in the first description. But in this description, unlike the first one where the momenta were determined by inspection, the analysis of constraints dictates the form of the expressions for the momenta.

To complete the equivalence between the two description at the Hamiltonian level, we need to recover the equation of motion from eqs $(30)$, by observing that one can re-write the first equation as

$$
\frac{\partial L_{0}}{\partial x}-\frac{d}{d t}\left(\frac{\partial L_{0}}{\partial q_{1}}\right)+\ddot{Q}_{1}=0
$$


then substituting in the second equation one gets

$$
\frac{\partial L_{0}}{\partial x}-\frac{d}{d t}\left(\frac{\partial L_{0}}{\partial q_{1}}\right)+\frac{d^{2}}{d t^{2}}\left(\frac{\partial L_{0}}{\partial q_{2}}\right)-\ddot{Q}_{2}=0
$$

Reiterating this procedure $m$ times yields the original equation of motion eq(3). With this we have shown that the new description is classically equivalent to that of Ostrogradsky description of higher order Lagrangians.

\section{Dirac brackets of the extended phase space variables}

In this section we are interested in computing Dirac brackets of the extended phase space degrees of freedom. Following the procedure we have described in the first section, one needs to determine the matrix of Poisson brackets of the constraints. Which in this case takes the simple form

$$
\Delta=\left(\begin{array}{cc}
0_{m} & I_{m} \\
-I_{m} & 0_{m}
\end{array}\right)
$$

but more relevant, is the inverse matrix

$$
\Delta^{-1}=\left(\begin{array}{cc}
0_{m} & -I_{m} \\
I_{m} & 0_{m}
\end{array}\right)
$$

Now we are in a position to compute all the relevant Dirac brackets, for instance

$$
\{x, P\}_{D}=\{x, P\}_{P}=1,
$$

while for the remaining degrees of freedom, they satisfy the following trivial algebra

$$
\begin{aligned}
& \left\{q_{i}, q_{j}\right\}=0 ;\left\{Q_{i}, Q_{j}\right\}=0 \\
& \left\{q_{i}, Q_{j}\right\}=0 .
\end{aligned}
$$

The last equation, eq(41), is expected since it suggests that the $q_{i}$ fields are non other than auxiliary fields. Furthermore, our calculation shows that the 
fundamental Poisson bracket of $(x, P)$ is not modified when generalised to Dirac bracket. Equipped with the above symplectic structure, the quantization of sysiems described by higher order Lagrangians becomes a trivial matter.

\section{Conclusion}

In the physics literature analysis of constraints is proven to be one of the viable but effective methods of establishing a Hamiltonian description of singular systems. Finite dynamical systems on compact spaces, QED, YangMills theories,....etc. have been a fertile land for such analysis.

In this paper we have shown that analysis of constraints provides a very natural Hamiltonian description of dynamical systems described by higher order Lagrangians. In fact this description has a very simple phase space structure. Consequently quantization of such systems, which is another publication in preparation, is easy to carry out.

\section{Acknowledgements}

The authors would like to thank Professor Abdus Salam, the International Atomic Energy Agency and UNESCO for hospitality at the International Centre for Theoretical Physics, Trieste. One of the authors (MSR) would also like to thank Professor J. Govaerts for useful discussions.

\section{References}

[1] A.Ostrogradsky, Me'langes de l' Acad. de St. Pe't. Oct. 1884.

[2] R. Abraham and J.E. Mardsen, Foundations of Mechanics, 2nd edition (Benjamin, Reading, Massachusetts, 1978); V.I. Arnold, Mathematical Methods of Classical Mechanics, (Springer-Verlag, New York, 1978). 
[3] E.C.G. Sudarshan and N. Mukunda, Classical Dynamics: a Modern Perspective (Jhone Wiley nd Sons, New York, 1974).

[4] P.A.M. Dirac, The Principles of Quantum Mechanics, 4th edition (Oxford Unoversity Press, 1985).

[5] P.A.M. Dirac, Lectures on Quantum Mechanics, (Belfer Graduate School of Science, Yeshiva University, New York, 1964).

[6] N.M.J. Woodhouse, Geometric Quantization (Oxford University Press, 1980); N.E. Hurt, Geometric Quantization in Action, (D. Reidel, Boston, 1983).

[7] A.J.Hanson, T. Regge and C. Teitelboim, Constrained Hamiltonian Systems (Academia Nazionale dei Lincei, Rome, 1976).

[8] K. Sundermeyer Constrained Dynamics, Lecture notes in Physics 169, (Springer-Virlag, Berlin, 1982).

[9] J. Govaerts, Hamiltonian Quantization and Constrained Dynamics, Leuven Notes in Mathematical and Theoretical Physica, (Leuven University Press, 1991). 
